\title{
Novel approaches to lipid-lowering therapy
}

\author{
D Brozin, ${ }^{1}$ MB BCh; F J Raal, ${ }^{2}$ FRCP, FRCPC, FCP (SA), Cert Endocrinology and Metabolism (SA) Phys, MMed, PhD \\ ${ }^{1}$ Department of Medicine, Faculty of Health Sciences, University of the Witwatersrand, Johannesburg, South Africa \\ ${ }^{2}$ Carbohydrate and Lipid Metabolism Research Unit and Division of Endocrinology and Metabolism, Department of Medicine, \\ Faculty of Health Sciences, University of the Witwatersrand, Johannesburg, South Africa
}

Corresponding author: F J Raal (frederick.raal@wits.ac.za)

\begin{abstract}
Cardiovascular disease (CVD) remains a major cause of death worldwide, with dyslipidaemia playing a significant role in the disease process. It is clinically useful to demarcate hypercholesterolaemia from hypertriglyceridaemia, with an increased serum low-density lipoprotein (LDL) cholesterol being the most powerful predictor of CVD morbidity and mortality, and a significant elevation in triglyceride levels increasing the risk of acute pancreatitis. Statins (with or without ezetimibe) and fibrates are the current first-line therapy in the management of dyslipidaemia. Although these medications have shown effectiveness in reducing CVD complications, there are patients who require a greater modification in lipid profile or are intolerant of first-line therapy. Novel agents are on the horizon, which have shown to lead to a significant decrease in serum LDL cholesterol. These include the proprotein convertase subtilisin/kexin type 9 (PCSK9) inhibitors (which have shown a reduction in CVD morbidity), mipomersen, cholesterol ester transfer protein (CETP) inhibitors and bempedoic acid. Further studies of the clinical benefit of these medications are ongoing. Drugs such as pemfibrate, angiopoietin-like protein 3 (ANGPTL3) inhibitors, apolipoprotein C3 (apo C3) inhibitors and diacylglycerol acyltransferase-1 (DGAT 1) inhibitors have shown promising results in the management of hypertriglyceridaemia. It is hoped that these exciting new technological advancements in the future management of dyslipidaemia will result in clinical benefit for patients.
\end{abstract}

S Afr Med J 2018;108(4):262-265. DOI:10.7196/SAMJ.2018.v108i4.13234

Cardiovascular disease (CVD) remains the major cause of death worldwide. In modern medicine, atherosclerotic CVD and its ensuing complications provide a significant challenge to clinicians, from the level of primary care to subspecialty practice. The role of dyslipidaemia in the pathogenesis of atherosclerotic CVD has been extensively researched, and doctors across South Africa (SA) are often tasked with modifying this significant risk factor burden among a wide variety of population groups.

When managing dyslipidaemia, it is clinically useful to separate hyperlipidaemia into two main categories, i.e. hypertriglyceridaemia and hypercholesterolaemia, particularly an increase in serum lowdensity lipoprotein (LDL) cholesterol concentrations. As discussed in this article, even though an elevated triglyceride (TG) level may result in an increased risk of CVD, the greatest effect of lowering markedly elevated serum TG $(>10 \mathrm{mmol} / \mathrm{L})$ is the reduction in the risk of developing acute pancreatitis, with a reduction of serum LDL cholesterol being a much more powerful predictor of risk reduction with regard to CVD morbidity and mortality.

\section{Management of predominant hypercholesterolaemia Statin therapy}

If diet and lifestyle advice does not lower LDL cholesterol sufficiently, the 3-hydroxy 3-methylglutaryl co-enzyme A (HMG-CoA) reductase inhibitors or statins are the current mainstay of treatment of elevated LDL cholesterol. The most efficacious statins at their maximum dose can reduce LDL cholesterol by up to $50 \%$.

\section{Ezetimibe}

Ezetimibe prevents intestinal absorption of cholesterol, and is currently the mainstay as a second-line agent in lowering LDL cholesterol. As per the European Society of Cardiology (ESC) guide- lines, ezetimibe is recommended as an add-on therapy for patients on high-intensity statins, who require further LDL cholesterol reduction, or for patients who are statin intolerant. The IMProved Reduction of Outcomes: Vytorin Efficacy International Trial (IMPROVE-IT), ${ }^{[1]}$ which showed a $2 \%$ absolute reduction in cardiovascular events compared with placebo over a 6-year follow-up period in patients on background statin therapy, has proven the benefit of the addition of ezetimibe to statin therapy in reducing cardiovascular events.

While these medications have been used to excellent effect in lowering the rate of complications secondary to dyslipidaemia, there are still certain groups of the population for whom this combination is insufficient. The two major groups of patients who may require an additional or alternative approach to lipid-lowering therapy, are: (i) individuals with familial hypercholesterolaemia, which is highly prevalent in the Afrikaner, Jewish and Indian populations of SA, as they require a greater percentage reduction in LDL cholesterol to achieve acceptable LDL cholesterol target levels; and (ii) individuals experiencing side-effects from statin therapy, precluding the use of high-intensity statin therapy, i.e. statin-intolerant patients.

\section{New therapies for the treatment of hypercholesterolaemia}

Proprotein convertase subtilisin/kexin type 9 inhibitor therapy Currently riding the crest of the wave with regard to lipid-lowering therapy, are the therapies that inhibit the action of the proprotein convertase subtilisin/kexin type 9 (PCSK9) enzyme. If PCSK9 binds to the LDL receptor (LDL-R), mainly on the hepatocyte, instead of being recycled to the cell surface, the LDL-R is degraded. By inhibiting the function of this enzyme through the use of monoclonal antibodies (mAbs), the expression of LDL-R on the hepatocyte is increased, thereby enhancing the cellular clearance of serum LDL cholesterol. As of 2018, alirocumab and evolocumab, two 
Table 1. Novel approaches to lipid lowering

\begin{tabular}{|c|c|c|c|c|}
\hline Class & Drug & LDL-C & Triglycerides & HDL-C \\
\hline HMG-CoA reductase inhibitors (statins) & $\begin{array}{l}\text { Atorvastatin, fluvastatin, lovastatin, } \\
\text { pravastatin, rosuvastatin, simvastatin, } \\
\text { pitavastatin* }\end{array}$ & $\downarrow \downarrow \downarrow$ & $\downarrow$ & $\uparrow$ \\
\hline PPAR alpha modulators & $\begin{array}{l}\text { Fibrates: bezafibrate, gemfibrozil, } \\
\text { fenofibrate, pemafibrate }^{*}\end{array}$ & $\downarrow$ & $\downarrow \downarrow \downarrow$ & $\uparrow$ \\
\hline Cholesterol absorption inhibitors & Ezetimibe & $\downarrow \downarrow$ & & \\
\hline PCSK9 inhibitors & $\begin{array}{l}\text { Alirocumab, evolocumab, LY3015014 } \\
\text { (mAbs), }{ }^{\star} \text { inclisiran (reduced synthesis) }{ }^{\star}\end{array}$ & $\downarrow \downarrow \downarrow$ & & \\
\hline Apo B100 inhibitors & Mipomersen* & $\downarrow \downarrow$ & & \\
\hline CETP inhibitors & Anacetrapib* & $\downarrow \downarrow$ & & $\uparrow$ \\
\hline ANGPTL3 inhibitors & $\begin{array}{l}\text { REGN1500* } \\
\text { Evinacumab* }\end{array}$ & $\downarrow$ & $\downarrow \downarrow \downarrow$ & $\downarrow$ \\
\hline Apo C3 inhibitors & Volanesorsen ${ }^{*}$ & $\downarrow$ & $\downarrow \downarrow \downarrow$ & \\
\hline Lp (a) inhibitors & IONIS-APO(a)Rx* & & & \\
\hline DGAT1 inhibitors & Pradigastat $^{*}$ & & $\downarrow \downarrow \downarrow$ & \\
\hline Triphosphate citrate lyase inhibitors & Bempedoic acid* & $\downarrow \downarrow$ & $\downarrow \downarrow$ & \\
\hline
\end{tabular}

mAbs against PCSK9, i.e. PCSK9 inhibitors, which are administered subcutaneously every 2 - 4 weeks, have received US Food and Drug Administration approval as lipid-lowering agents. ${ }^{[2]}$ Unfortunately, they have not yet been approved for clinical use in SA. These exciting new medications have been shown by numerous clinical trials to produce a significant reduction in serum LDL cholesterol of $\sim 60 \%$ when used either as monotherapy or when combined with a statin with or without ezetimibe. ${ }^{[3]}$ The recently published Further Cardiovascular Outcomes Research with PCSK9 Inhibition in Subjects with Elevated Risk (FOURIER) trial ${ }^{[4]}$ investigated cardiovascular outcomes in high-risk patients on background statin therapy, who were receiving evolocumab v. placebo and showed not only a significant reduction in serum LDL cholesterol concentration, but also a significant reduction in cardiovascular morbidity in those receiving the PCSK9 inhibitor. A similar study with alirocumab, the Evaluation of Cardiovascular Outcomes After an Acute Coronary Syndrome During Treatment With Alirocumab (ODYSSEY Outcomes), ${ }^{[5]}$ has recently been completed and the results are eagerly awaited. A third monoclonal against PCSK9, LY3015014, is currently being developed. Current studies show that the reduction of serum LDL cholesterol concentration with the use of LY3015014 is comparable to levels achieved by the mAbs now on the market. ${ }^{[5]}$ The advantage of this $\mathrm{mAb}$, compared with the current medication available, is its prolonged half-life, which enables patients to enter into a regimen requiring subcutaneous administration of the drug at 4 - 8-weekly intervals, as opposed to the current $\mathrm{mAb}$ regimen, which is administered at $2-4$-weekly intervals.

Regarding the modulation of PCSK9 function, a fourth drug, inclisiran, is currently undergoing phase 3 clinical trials, and is showing promising results with regard to lowering serum LDL cholesterol concentrations. ${ }^{[6]}$ As opposed to the mAbs, inclisiran reduces the activity of PCSK9 by inhibiting synthesis of the enzyme, acting at the level of protein translation via RNA interference in the liver. Unlike mAbs, inclisiran only needs to be administered once every 3 - 6 months. However, a cardiovascular outcome study with inclisiran will be required to prove that it has similar cardiovascular benefit to PCSK9 mAb therapy.

\section{Mipomersen}

A major component in the hepatic synthesis of very low-density lipoprotein (VLDL) and hence LDL, is the transcription and translation of apolipoprotein B-100 (apo B100), the carrier protein of LDL. A novel agent, mipomersen, which is not yet available in SA, inhibits the synthesis of apo B100 by ribonucleic acid (RNA) interference at the transcription stage. In doing so, this medication, classed as an antisense oligonucleotide (ASO), can reduce serum LDL cholesterol using an alternate pathway than the statins and PCSK9 inhibitors. Mipomersen is administered as a weekly subcutaneous injection. Studies ${ }^{[7]}$ with mipomersen have shown that this therapy can produce a significant reduction in both serum LDL cholesterol (25 - 39\%), as well as lipoprotein (a) [Lp (a)] (21 - $39 \%$ ) when added to standard treatment regimens. There are some data to suggest that mipomersen may also result in a reduction in cardiovascular morbidity ${ }^{[8]}$ The largest barriers with regard to mipomersen are injection site reactions and the propensity for the development of fatty liver (hepatic steatosis), although the long-term morbidity and mortality with regard to hepatic disease with this agent are yet to be studied.

\section{Cholesterol ester transfer protein inhibitors}

Cholesterol ester transfer protein (CETP) is an important mediator protein in the lipid transport cycle owing to its ability to transfer cholesterol esters between lipoproteins. Inhibition of CETP results in a marked increase in serum high-density lipoprotein cholesterol (HDL cholesterol), with a reduction in serum LDL cholesterol. Three medications developed against CETP, i.e. torcetrapib, dalcetrapib and evacetrapib, have been abandoned in the trial stage owing to either adverse events or lack of cardiovascular benefit, with only anacetrapib still undergoing further evaluation. The recently reported Randomized Evaluation of the Effects of Anacetrapib through Lipid Modification (REVEAL) ${ }^{[9]}$ trial showed a modest reduction in cardiovascular events, but the reduction was proportional to the reduction in LDL cholesterol and not related to the marked increase in HDL cholesterol observed with anacetrapib. It is therefore unlikely that CETP inhibitors will undergo further clinical development. 


\section{Management of predominant hypertriglyceridaemia}

Dietary change and weight loss in patients who are overweight or obese can effectively reduce serum TG levels. However, if diet and lifestyle do not lower TG levels sufficiently, drug therapy may be required.

Statins can modestly reduce serum TG levels and are the agents of choice for mild hypertriglyceridaemia (TG $<5 \mathrm{mmol} / \mathrm{L}$ ), particularly if the LDL cholesterol is also elevated. However, when TG levels are markedly elevated, particularly if $>10 \mathrm{mmol} / \mathrm{L}$, there is a risk of developing acute pancreatitis, which is potentially life threatening. In such cases, additional or alternative TG-lowering therapies may be required. The major classes of drugs used for the treatment of hypertriglyceridaemia are the fibrates and niacin (nicotinic acid). However, niacin has been withdrawn in many countries globally, including SA, because of side-effects or lack of cardiovascular benefit.

\section{Fibrates and pemafibrate}

Fibrinic acid derivatives, or fibrates, are currently available in SA as first-line treatment for patients with severe hypertriglyceridaemia. These agents are agonists of the nuclear protein peroxisome proliferator activated receptor alpha (PPAR alpha) and therefore stimulate the synthesis of HDL and the activity of lipoprotein lipase, resulting in the increased hydrolysis of TGs, as well as a reduction in synthesis of apo C3. These agents are an important therapy for the prevention of acute pancreatitis and its clinical complications in patients with severe hypertriglyceridaemia $(>10 \mathrm{mmol} / \mathrm{L})$. Currently, there are no robust outcome studies that have demonstrated a definitive reduction in CVD morbidity and mortality with fibrate therapy. However, a reduction in CVD events was noted in a subgroup analysis of patients with high TG levels and low HDL cholesterol. ${ }^{[10]}$ Fibrates, particularly fenofibrate and bezafibrate, are well tolerated and can be used in combination with statin therapy in patients requiring a reduction in both serum LDL cholesterol and TG levels.

Pemafibrate is a new drug to the fibrate class and reveals a greater selectivity and activity of the PPAR alpha protein. This agent has been shown to produce a significant reduction in serum TG levels and is currently under investigation for its cardiovascular benefit in a large outcome study, the Pemafibrate to Reduce Cardiovascular OutcoMes by Reducing Triglycerides IN patiENts With diabeTes (PROMINENT) ${ }^{[11]}$ which is enrolling patients with a combination of a high serum TG and low HDL cholesterol. If significant results are obtained, pemafibrate may be an interesting alternative to currently available fibrate therapy.

\section{Angiopoietin-like protein 3 inhibitors}

Other medications belonging to the classes of antisense oligonucleotides are those that inhibit the production of the angiopoietin-like protein 3 (ANGPTL3) enzyme. The enzyme, ANGPTL3, has been shown to inhibit lipoprotein lipase (LPL) and hence its modulation results in a reduction of serum TG. An interesting finding is that the inhibition of ANGPTL3 not only results in the reduction of serum TG, as expected, by reduction of LPL activity, but also in a reduction of serum LDL cholesterol. ${ }^{[12]}$ The mechanism by which this reduction of serum LDL cholesterol is achieved is unknown. A second approach with regard to the inhibition of ANGPTL3 is the development of a monoclonal antibody against the enzyme. This $\mathrm{mAb}$, evinacumab, has been shown to reduce TGs, LDL cholesterol and Lp (a) and is therefore an exciting new avenue for further exploration in the quest to lower lipids and improve cardiovascular outcomes.

\section{Apo C3 inhibitors}

Two recently published studies have shown the powerful TG-lowering effect that can be produced by the inhibition of apo C3. These studies, i.e. a Study of Volanesorsen (formerly IONIS-APOCIIIRx) in Patients With Familial Chylomicronemia Syndrome (APPROACH) and the Cardiovascular Outcomes for People Using Anticoagulation Strategies (COMPASS) trial, ${ }^{[13]}$ have shown a serum TG reduction of up to $50 \%$ with the use of volanesorsen, an apo C3 inhibitor, when used as monotherapy in the management of dyslipidaemia. By inhibiting the apo C3 gene, there is both enhanced activity of LPL, resulting in an increased TG clearance, as well as a reduction in the synthesis of VLDL. The major limiting factor with the use of volanesorsen was the side-effects related to its use, i.e. severe injection site reactions and thrombocytopenia. Further studies investigating the clinical benefit of these medications against the side-effect profile are yet to be conducted.

\section{Lipoprotein (a) inhibitors}

Lp (a), a particle with structural similarity to plasminogen, has been shown to be an independent risk factor for CVD, as well as for the development of aortic stenosis. This is probably because Lp (a) is an LDL-like particle, but it is also thought to inhibit the action of plasminogen, resulting in a reduction in fibrinolysis; hence, it is both proatherogenic and prothrombotic. An antisense oligonucleotide, IONIS-APO(a)Rx, is currently undergoing clinical trials, with preliminary results showing a significant reduction in Lp (a) levels with the use of this treatment. ${ }^{[14]}$ The use of this medication in lowering Lp (a) and subsequent CVD is yet to be determined, but it provides an interesting alternate pathway in lowering cardiovascular mortality in patients requiring Lp (a) reduction therapy.

\section{Other novel therapies on the horizon}

Pradigastat is an oral inhibitor of the enzyme diacylglycerol acyltransferase 1 (DGAT1), which prevents fat absorption in the small intestine, as well as TG synthesis. This drug is currently undergoing large-scale clinical trials, with small studies revealing a serum TG reduction of up to $70 \% \cdot{ }^{[15]}$

Bempedoic acid (ETC-1002) is currently undergoing phase 2 clinical trials, with early results showing a significant reduction in LDL cholesterol, $30 \%$ as monotherapy and $50 \%$ when added to ezetimibe. ${ }^{[16]}$ This drug inhibits the enzyme adenosine triphosphate citrate lyase, which results in a decreased production of both cholesterol and TGs.

In newer studies, the role of modulation of the receptor asialoglycoprotein receptor 1 (ASGR1) is being explored. Individuals with a genetic variant of ASGR1 have been shown to have a lower LDL cholesterol, lower TG and higher HDL cholesterol concentrations. Based on this finding, it may be of interest to explore this receptor, and hence the modulation of its function, to produce a favourable outcome in patients with dyslipidaemia.

\section{Conclusion}

Treatment modalities in the management of dyslipidaemia and the complications thereof are making tremendous leaps forward as medical technology advances. These new and novel approaches are now widening the net for individuals who require a greater reduction in serum lipid concentrations, and are also showing promise in patients who were previously unable to partake in first-line therapies owing to side-effects. The end goal that will hopefully be achieved with these exciting new strategies is to lower patients' risk profile, while improving compliance by enhancing dose scheduling and reducing the side-effect profile of these treatments. It is our hope 
that the thirst for knowledge in combating dyslipidaemia will lead to a continued push toward technological advancement in this exciting field of medicine.

Acknowledgements. None.

Author contributions. Both authors contributed equally to this review.

Funding. None.

\section{Conflicts of interest. None.}

1. Phan BA, Dayspring TD, Toth PP. Ezetimibe therapy: Mechanism of action and clinical update. Vasc Health Manag 2012;2012(8):415-427. https://doi.org/10.2147/VHRM.S33664

2. Stein EA, Raal FJ. New therapies for reducing low density lipoprotein cholesterol. Endocrinol Metab Clin N Am 2014;43(4):1007-1033. https://doi.org/10.1016/j.ecl.2014.08.00

3. Ray KK, Ginsberg HN, Davidson MH, et al. Reductions in atherogenic lipids and major cardiovascular events: A pooled analysis of 10 ODYSSEY trials comparing alirocumab with control. Circulation 2016;134(4):1931-1943. https://doi.org/10.1161/circulationaha.116.024604

4. Navarese EP, Kolodzietjczak M, Schulze V, et al. Effects of proprotein convertase subtilisin/kexin type 9 antibodies in adults with hypercholesterolemia: A systemic review and meta analysis. Ann Intern Med 2015;163(1):40-51. https://doi.org/10.7326/m14-2957

5. Kastelein JJ, Nissen SE, Rader DJ, et al. Safety and efficacy of LY3015014, a monoclonal antibody to proprotein convertase subtilisin/kexin type 9 (PCSK 9): A randomized placebo controlled phase study. Eur Heart J 2016;37(17):1360-1369. https://doi.org/10.1093/eurhearti/ehv707

6. Fitzgerald $\mathrm{K}$, White S, Borodovsky A, et al. A highly durable RNAi therapeutic inhibitor of PCSK9. 6. Fitzgerald K, White S, Borodovsky A, et al. A highly durable RNAi theraps
7. Santos RD, Raal FJ, Catapino Al, et al. Mipomersen, an oligonucleotide to apolipoprotein B-100, reduces lipoprotein(a) in various populations with hypercholesterolemia: Results of 4 phase 3 trials. Arterioscler Thromb Vasc Biol 2015;35(3):689-699. https://doi.org/10.1161/atvbaha.114.304549

8. Duell PB, Santos RD, Kirwan B-A, et al. Long term mipomersen treatment is associated with a reduction in cardiovascular events in patients with familial hypercholesterolemia. J Clin Lipidol 016;10(4):1011-1021. https://doi.org/10.1016/j.jacl.2016.04.013

9. HPS3/TIMI55-REVEAL Collaborative group. Effects of anacetrapib in patients with atherosclerotic vascular disease. N Engl J Med 2017;377(13):1217-1227. https://doi.org/10.1056/nejmoal706444

. Jun M, Foote C, Lv J, et al. Effects of fibrates on cardiovascular outcomes: A systematic review and meta-analysis. Lancet 2010;375(9729):1875-1884. https://doi.org/10.1016/S0140-6736(10)60656-3

11. Keech AC, Jenkins AJ. Triglyceride lowering therapies. Curr Opin Lipidol 2017;28(6):477-487. https:// doi.org $/ 10.1097 / \mathrm{mol} .0000000000000465$

2. Tikka A, Juahianinen M. The role of ANGPTL3 in controlling lipoprotein metabolism. Endocrine 2016;52(2):187-193. https://doi.org/10.1007/s12020-015-0838-9

13. Cupido AJ, Reeskamp LF, Kastelein JP. Novel lipid modifying drugs to lower LDL cholesterol. Curr Opin Lipidol 2017;28(4):367-373. https://doi.org/10.1097/mol.0000000000000428

14. Tsimikas S, Viney NJ, Hughes SG, et al. Antisense therapy targeting apolipoprotein(a); a randomized double blind, placebo controlled phase 1 study. Lancet 2015;386(10002):1472-1483. https:///doi.org/10.1016/s01406736(15)61252-1

15. Meyers CD, Tremblay K, Amer A, et al. Effect of DGAT1 inhibitor pradigastat on triglyceride and apoB48 levels in patients with familial chylomicronemia syndrome. Lipids Health Dis 2015;14(1):8. https://doi.org/10.1186/s12944-015-0006-5

16. Thompson PD, MacDougall DE, Newton RS, et al. Treatment with ETC-1002 alone and in combination with ezetimibe lowers LDL cholesterol in hypercholesterolemic patients with or without statin intolerance. J Clin Lipidol 2016;10(3):556-567. https://doi.org/10.1016/j.jacl.2015.12.025

Accepted 5 March 2018 